Supplemental Information

\title{
Intestinal excretion, intestinal recirculation and renal tubule reabsorption are under-appreciated mechanisms that drive the distribution and pharmacokinetic behavior of small molecule drugs
}

Donglu Zhang*, Cong Wei, Cornelis ECA Hop, Matthew R Wright, Ming Hu, Yurong Lai, S Cyrus Khojasteh, W Griffith Humphreys

Corresponding author: Donglu Zhang, Drug Metabolism and Pharmacokinetics, Genentech, 1 DNA Way, South San Francisco, CA 94080, zhang.donglu@gene.com, Phone 650-291-0058

Table S1. Pharmacokinetic parameters of digoxin and nitrofurantoin following single IV dose of radiolabels to male bile-cannulated Sprague-Dawley rats $(\mathrm{WT}=$ wild-type) and $\mathrm{P}-\mathrm{gp}-\mathrm{KO}$ or BCRP-KO rats

Table S2. Intestinal clearance, renal clearance, and biliary clearance of apixaban, digoxin, and nitrofurantoin in Sprague-Dawley rats

Table S3. Study designs in dogs and rats following oral and intravenous administration of apixaban, $\left[{ }^{14} \mathrm{C}\right]$ apixaban, $\left[{ }^{14} \mathrm{C}\right]$ digoxin, and $\left[{ }^{14} \mathrm{C}\right]$ nitrofurantoin with and without oral administration of activated charcoal ${ }^{13}$

Table S4. Percent recoveries of radioactivity ( \pm SD) following a single IV dose of $\left[{ }^{3} \mathrm{H}\right]$ digoxin, $\left[{ }^{14} \mathrm{C}\right]$ nitrofurantoin, or $\left[{ }^{14} \mathrm{C}\right]$ apixaban to male bile-cannulated SpragueDawley rats $(\mathrm{WT}=\text { wild-type; } \mathrm{P} \text {-gp-KO }=\mathrm{P} \text {-gp-KO; } \mathrm{BCRP}-\mathrm{KO}=\mathrm{BCRP}-\mathrm{KO})^{3}$ following a single $1 \mathrm{mg} / \mathrm{kg}$ IV dose of $\left[{ }^{14} \mathrm{C}\right]$ apixaban to male intact and bile-cannulated Beagle dogs ${ }^{13}$ Table S5. Classes of drugs that are substrates of intestinal efflux transporters

Table S6. Selected studies that tested the effects of activated charcoal on pharmacokinetics and pharmacodynamics of drugs

Figure S1. Chemical structures used in Table S5

Additional References 
Table S1. Pharmacokinetic parameters of digoxin and nitrofurantoin following single IV dose of radiolabels to male bile-cannulated Sprague-Dawley rats (WT = wild-type) and P-gp-KO or BCRP-KO rats

\begin{tabular}{|c|c|c|c|c|}
\hline & \multicolumn{2}{|c|}{$\begin{array}{c}{\left[{ }^{3} \mathrm{H}\right] \text { Digoxin }(1 \mathrm{mg} / \mathrm{kg})} \\
(\mathrm{n}=3 \mathrm{BDC})\end{array}$} & \multicolumn{2}{|c|}{$\begin{array}{c}{\left[{ }^{14} \mathrm{C}\right] \text { Nitrofurantoin Dose }(5 \mathrm{mg} / \mathrm{kg})} \\
(\mathrm{n}=3 \text { BDC })\end{array}$} \\
\hline & WT Control & P-gp-KO & WT Control & BCRP-KO \\
\hline $\operatorname{AUC}_{0-12}(\boldsymbol{\mu g} \cdot \mathbf{h} / \mathbf{m L})$ & $1.12 \pm 0.31$ & $1.13 \pm 0.27$ & $1.23 \pm 0.08$ & $1.68 \pm 0.27$ \\
\hline$C_{\max }(\mu \mathrm{g} / \mathrm{mL})^{1}$ & $1.30 \pm 0.07$ & $1.35 \pm 0.18$ & $3.48 \pm 0.26$ & $4.03 \pm 0.63$ \\
\hline$C_{12}(n g / m L)$ & $5.0 \pm 4.1$ & $2.6 \pm 2.1$ & $2.2 \pm 0.4$ & $1.2 \pm 0.3$ \\
\hline$T_{1 / 2}(h)$ & $2.4 \pm 1.1$ & $1.9 \pm 0.1$ & $1.5 \pm 0.8$ & $0.9 \pm 0.4$ \\
\hline CL $(\mathrm{mL} / \mathrm{min} / \mathrm{kg})$ & $16 \pm 4.2$ & $15 \pm 3.3$ & $65 \pm 4$ & $50 \pm 8.3$ \\
\hline Vss (L/kg) & $1.3 \pm 0.3$ & $1.2 \pm 0.2$ & $1.6 \pm 0.2$ & $1.1 \pm 0.1$ \\
\hline MRT (h) & $1.6 \pm 0.8$ & $1.4 \pm 0.4$ & $0.4 \pm 0.1$ & $0.4 \pm 0.0$ \\
\hline$C \mathbf{L}_{\text {IE }}(\mathrm{L} / \mathbf{h} / \mathbf{k g})$ & 1.6 & 0.1 & 0.3 & 0.1 \\
\hline$C L_{R}(\mathrm{~mL} / \mathrm{min} / \mathrm{kg})^{2,3}$ & 5.5 & 3.5 & 32.6 & 20.6 \\
\hline$C L_{b}(\mathrm{~mL} / \mathrm{min} / \mathrm{kg})$ & 2.1 & 1.1 & 1.2 & 0.5 \\
\hline$C L_{\text {met }}(\mathrm{mL} / \mathrm{min} / \mathrm{kg})$ & 6.8 & 10.3 & 30.9 & 28.8 \\
\hline
\end{tabular}

${ }^{1}$ The plasma concentration at 5 min.

${ }^{2}$ The protein binding of digoxin and nitofurantoin in rat plasma was $20 \%$, therefore, GFRxfu $=0.31 \times 0.20=1.1 \mathrm{~mL} / \mathrm{min} / \mathrm{kg}$ for digoxin and GFRxfu $=0.31 \times 0.40=2.2 \mathrm{~mL} / \mathrm{min} / \mathrm{kg}$ for nitrofurantoin.

${ }^{3}$ Unchanged $\left[{ }^{14} \mathrm{C}\right]$ digoxin accounted for $>95,90,36$, and $88 \%$ in wild-type rats and $>95,78,12$, and $81 \%$ in P-gp-knockout rats of total radioactivity in plasma, urine, bile, and feces, respectively. Unchanged $\left[{ }^{14} \mathrm{C}\right]$ nitrofurantoin accounted for $>90,72,30$, and $4 \%$ in wild-type rats and $>90,58,9$, and 3.5\% in BCRP-knokout rats of total radioactivity in plasma, urine, bile, and feces, respectively. 
Table S2. Intestinal clearance, renal clearance, and biliary clearance of apixaban, digoxin, and nitrofurantoin in Sprague-Dawley rats

\begin{tabular}{|c|c|c|c|}
\hline Drug Properties & Apixaban & Digoxin & Nitrofurantoin \\
\hline MW & 446 & 780 & 238 \\
\hline Solubility & Soluble & Soluble & Soluble \\
\hline Apparent permeability $(\mathrm{nm} / \mathrm{S})$ & $>200$ & $<1$ & $<1$ \\
\hline Protein binding & $93 \%$ & $80 \%$ & $60 \%$ \\
\hline Substrate of transporters & P-gp and BCRP & P-gp, OATP2B1 & BCRP \\
\hline Intestinal clearance $(\mathrm{mL} / \mathrm{min} / \mathrm{kg}), \%$ of total & $5.0(21 \%)$ & $1.6(10 \%)$ & $0.3(0.5 \%)$ \\
\hline Renal clearance $(\mathrm{mL} / \mathrm{min} / \mathrm{kg}), \%$ of total & $11.8(51 \%)$ & $5.5(35 \%)$ & $32.6(50 \%)$ \\
\hline Biliary Clearance $(\mathrm{mL} / \mathrm{min} / \mathrm{kg}), \%$ of total & $0.3(1 \%)$ & $2.1(13 \%)$ & $1.2(2 \%)$ \\
\hline Metabolism clearance $(\mathrm{mL} / \mathrm{min} / \mathrm{kg}), \%$ of total & $6.2(27 \%)$ & $6.8(42 \%)$ & $30.9(48 \%)$ \\
\hline
\end{tabular}


Table S3. Study designs in dogs and rats following oral and intravenous administration of apixaban, $\left[{ }^{14} \mathrm{C}\right]$ apixaban, $\left[{ }^{14} \mathrm{C}\right]$ digoxin, and $\left[{ }^{14} \mathrm{C}\right]$ nitrofurantoin with and without oral administration of activated charcoal ${ }^{13}$

\begin{tabular}{|c|c|c|c|c|c|}
\hline Test Article & Species (n) & $\begin{array}{l}\text { Dosing } \\
\text { Routes }\end{array}$ & $\begin{array}{l}\text { Other } \\
\text { Treatments }\end{array}$ & Dose (mg/kg) & Sample Collection \\
\hline Apixaban & $\operatorname{Dog}(4)$, intact & $\mathrm{PO}$ & $\begin{array}{l}\text { No, Charcoal at } \\
0.25,1 \text {, and } 3 \mathrm{~h}\end{array}$ & 5 & $\begin{array}{l}\text { Plasma }(0.5,1,2,4,6,8,12,14,20 \text {, } \\
24 \mathrm{~h})\end{array}$ \\
\hline$\left[{ }^{14} \mathrm{C}\right]$ Apixaban & $\operatorname{Dog}(3)$, Intact & $\mathrm{IV}^{5}$ & No & 1 & Urine, bile, feces, $(0-72 \mathrm{~h})$ \\
\hline$\left[{ }^{14} \mathrm{C}\right]$ Apixaban & $\operatorname{Dog}(2), \mathrm{BDC}$ & $\mathrm{IV}^{5}$ & No, Charcoal $^{3}$ & 1 & Plasma $^{1}$, urine, bile, feces, $(0-72 \mathrm{~h})$ \\
\hline Apixaban & Rat(3), WT, intact & $\mathrm{PO}$ & No & 4 & Plasma $(0.5,1,2,3,4,5,7,10,24)$ \\
\hline Apixaban & Rat(3), WT, intact & PO & Elacridar, $-1 \mathrm{~h}$ & 4 & Plasma $(0.5,1,2,3,4,5,7,10,24)$ \\
\hline Apixaban & Rat(3), Pgp-KO, intact & PO & No & 4 & Plasma $(0.5,1,2,3,4,5,7,10,24)$ \\
\hline Apixaban & $\operatorname{Rat}(3), \mathrm{BCRP}-\mathrm{KO}$, intact & $\mathrm{PO}$ & No & 4 & Plasma $(0.5,1,2,3,4,5,7,10,24)$ \\
\hline$\left[{ }^{14} \mathrm{C}\right]$ Apixaban & Rat(3), WT, intact & IV & No & 2 & Urine, bile, feces, $(0-24 \mathrm{~h})$ \\
\hline$\left[{ }^{14} \mathrm{C}\right]$ Apixaban & Rat(3), WT, BDC & IV & No & 2 & Plasma $^{2}$, urine, bile, feces, $(0-48$ h) \\
\hline$\left[{ }^{14} \mathrm{C}\right]$ Apixaban & $\operatorname{Rat}(3), \mathrm{WT}, \mathrm{BDC}$ & IV & Elacridar, $-1 / 8 \mathrm{~h}$ & 2 & Plasma $^{2}$, urine, bile, feces, $(0-48 \mathrm{~h})$ \\
\hline$\left[{ }^{14} \mathrm{C}\right]$ Apixaban & Rat(3), WT, BDC & IV & Charcoal $^{4}$ & 2 & Plasma $^{2}$, urine, bile, feces, $(0-48$ h) \\
\hline$\left[{ }^{14} \mathrm{C}\right]$ Apixaban & $\operatorname{Rat}(3)$, P-gp-KO, BDC & IV & No & 2 & Plasma $^{2}$, urine, bile, feces, $(0-48 \mathrm{~h})$ \\
\hline$\left[{ }^{14} \mathrm{C}\right]$ Apixaban & $\operatorname{Rat}(3), \mathrm{BCRP}-\mathrm{KO}, \mathrm{BDC}$ & IV & No & 2 & Plasma $^{2}$, urine, bile, feces, $(0-48 \mathrm{~h})$ \\
\hline$\left[{ }^{3} \mathrm{H}\right]$ Digoxin & Rat (3), WT, BDC & IV & No & 1 & Plasma, urine, bile, feces, $(0-48 \mathrm{~h})$ \\
\hline$\left[{ }^{3} \mathrm{H}\right]$ Digoxin & Rat (3), P-gp-KO, BDC & IV & No & 1 & Plasma, urine, bile, feces, $(0-48 \mathrm{~h})$ \\
\hline$\left[{ }^{14} \mathrm{C}\right]$ Nitrofurantoin & Rat (3), WT, BDC & IV & No & 5 & Plasma, urine, bile, feces, $(0-48 \mathrm{~h})$ \\
\hline$\left[{ }^{14} \mathrm{C}\right]$ Nitrofurantoin & Rat (3), P-gp-KO, BDC & IV & No & 5 & Plasma, urine, bile, feces, $(0-48 \mathrm{~h})$ \\
\hline
\end{tabular}

${ }^{1}$ Time points: $0.083,0.25,0.5,1,2,3,5,8,24 \mathrm{~h} ;{ }^{2}$ Time points: $0.083,0.25,1,2,4,6,8,12,24 \mathrm{~h} ;{ }^{3}$ Charcoal (via gavage) dosing time: $10,90,180$, $420 \mathrm{~min} ;{ }^{4}$ Charcoal (via intraduodenal route) dosing times: $-30,5,90,180 \mathrm{~min} ;{ }^{5} \mathrm{IV}$ infusion time: 5 min. 
Table S4. Percent recoveries of radioactivity $( \pm \mathrm{SD})$ following a single IV dose of $\left[{ }^{3} \mathrm{H}\right]$ digoxin, $\left[{ }^{14} \mathrm{C}\right]$ nitrofurantoin, or $\left[{ }^{14} \mathrm{C}\right]$ apixaban to male bile-cannulated Sprague-Dawley rats (WT = wild-type; P-gp-KO = P-gp-KO; BCRP-KO = BCRP-KO) ${ }^{3}$ following a single 1 $\mathrm{mg} / \mathrm{kg}$ IV dose of $\left[{ }^{14} \mathrm{C}\right]$ apixaban to male intact and bile-cannulated Beagle dogs ${ }^{13}$

\begin{tabular}{|c|c|c|c|c|c|}
\hline \multirow[t]{2}{*}{ Compound } & \multirow[t]{2}{*}{ Animals } & \multicolumn{4}{|c|}{ Dose Recovery (\% of dose in 0-24 h Collections) } \\
\hline & & Urine & Bile & Feces & Total \\
\hline & Rat & & & & \\
\hline$\left[{ }^{14} \mathrm{C}\right]$ Apixaban (IV) & WT & $49.5 \pm 4.2$ & $10.7 \pm 1.1$ & $21.1 \pm 5.2$ & $82.3 \pm 2.2$ \\
\hline $\begin{array}{c}{\left[{ }^{14} \mathrm{C}\right] \text { Apixaban (IV) and GF- }} \\
120918\end{array}$ & WT & $50.6 \pm 3.1$ & $14.7 \pm 2.2$ & $13.8 \pm 2.4$ & $79.1 \pm 2.5$ \\
\hline$\left[{ }^{14} \mathrm{C}\right]$ Apixaban (IV) and Charcoal & WT & $40.7 \pm 2.6$ & $15.7 \pm 1.9$ & $28.8 \pm 2.5$ & $85.2 \pm 5.9$ \\
\hline$\left[{ }^{14} \mathrm{C}\right]$ Apixaban (IV) & Pgp-KO & $41.7 \pm 5.5$ & $11.4 \pm 4.1$ & $26.1 \pm 3.7$ & $79.2 \pm 5.6$ \\
\hline$\left[{ }^{14} \mathrm{C}\right]$ Apixaban (IV) & BCRP-KO & $27.3 \pm 15.3$ & $15.2 \pm 3.1$ & $39.7 \pm 9.6$ & $82.1 \pm 2.6$ \\
\hline$\left[{ }^{14} \mathrm{C}\right]$ Apixaban (IV) ${ }^{1}$ & WT-intact & $51.3 \pm 5.1$ & NA & $41.1 \pm 1.9$ & $92.4 \pm 3.2$ \\
\hline$\left[{ }^{14} \mathrm{C}\right]$ Apixaban (oral) ${ }^{2}$ & Intact & 13.4 & NA & 74.0 & 87.4 \\
\hline$\left[{ }^{14} \mathrm{C}\right]$ Apixaban $(\mathrm{PO})^{2}$ & $\mathrm{BDC}$ & 10.5 & 2.6 & 69.8 & 82.9 \\
\hline$\left[{ }^{3} \mathrm{H}\right]$ Digoxin (IV) & WT & $41.9 \pm 5.1$ & $38.8 \pm 8.1$ & $12.0 \pm 1.2$ & $92.7 \pm 2.1$ \\
\hline$\left[{ }^{3} \mathrm{H}\right]$ Digoxin (IV) & Pgp-KO & $30.0 \pm 7.4$ & $61.9 \pm 11.7$ & $1.2 \pm 0.4$ & $93.1 \pm 4.0$ \\
\hline$\left[{ }^{14} \mathrm{C}\right]$ Nitrofurantoin (IV) & WT & $66.9 \pm 3.5$ & $5.7 \pm 1.3$ & $10.6 \pm 0.6$ & $83.2 \pm 2.6$ \\
\hline \multirow[t]{2}{*}[{}^{14}\mathrm{C}]{ Nitrofurantoin (IV) } & $\mathrm{BCRP}-\mathrm{KO}$ & $71.5 \pm 6.0$ & $7.4 \pm 2.5$ & $4.9 \pm 2.3$ & $83.8 \pm 6.1$ \\
\hline & Dog & & & & \\
\hline$\left[{ }^{14} \mathrm{C}\right]$ Apixaban $(\mathrm{IV})^{3}$ & $\mathrm{BDC}$ & $13.5,19.1$ & $29.4,12.4$ & $42.7,48.6$ & $85.6,80.1$ \\
\hline$\left[{ }^{14} \mathrm{C}\right]$ Apixaban (IV) and Charcoal & $\mathrm{BDC}$ & $16.3,8.1$ & $20.5,13.1$ & $62.7,74.4$ & $99.5,95.6$ \\
\hline
\end{tabular}




\begin{tabular}{|c|c|c|c|c|c|}
\hline$\left[{ }^{14} \mathrm{C}\right]$ Apixaban (IV) & Intact & $9.9 \pm 7.9$ & NA & $50.8 \pm 8.3$ & $60.7 \pm 10.6$ \\
\hline$\left[{ }^{14} \mathrm{C}\right]$ Apixaban $(\mathrm{PO})^{4}$ & Intact & 8.8 & $\mathrm{NA}$ & 73.7 & 82.5 \\
\hline
\end{tabular}

${ }^{1}$ Collections of $0-48 \mathrm{~h}$.

${ }^{2} \mathrm{PO}$ dose was $30 \mathrm{mg} / \mathrm{kg}$ and collections were $0-168 \mathrm{~h}$ for 3 intact and $0-48 \mathrm{~h}$ for $3 \mathrm{BDC}$ rats and listed here for comparison.

${ }^{3}$ A crossover study with dosing separated by one week. ${ }^{13}$

${ }^{4} \mathrm{PO}$ dose was $5 \mathrm{mg} / \mathrm{kg}$ and collections were $0-168 \mathrm{~h}$ for 3 intact dogs and listed here for comparison. ${ }^{13}$

${ }^{5}$ In dogs, unchanged $\left[{ }^{14} \mathrm{C}\right]$ apixaban accounted for $>95,83$, and $81 \%$ of total radioactivity in plasma, urine and feces. In rats, unchanged

$\left[{ }^{14} \mathrm{C}\right]$ apixaban accounted for $96.4,93.7,91.2 \%$ in urine, $20.7,34.8$, and $18 \%$ in bile, $74,79.6$, and $87.2 \%$ in feces of wild-type, $\mathrm{P}$-gp-KO, and BCRP-KO rats, respectively. 
Table S5. Classes of drugs that are substrates of intestinal efflux transporters. Study Methods describe experimental approaches to determine if a compound is a substrate of an efflux transporter

\begin{tabular}{|c|c|c|c|c|}
\hline Class of Drug & $\begin{array}{c}\text { Major } \\
\text { Transporters }\end{array}$ & Selected Drugs & Study Methods & References \\
\hline Anticoagulants & BCRP, P-gp & Apixaban, rivaroxaban, edoxaban & $\begin{array}{l}\text { In vitro, transporter } \\
\text { knockout }\end{array}$ & $\begin{array}{l}\text { This study, (Gnoth et al., } \\
2011)^{120}\end{array}$ \\
\hline Antiepileptics & P-gp & $\begin{array}{l}\text { Phenytoin, phenobarbital, lamotrigine, levetiracetam, } \\
\text { gabapentin, felbamate }\end{array}$ & In vitro permeability & $\begin{array}{l}\text { (Loscher and Potschka, } \\
\text { 2005; Luna-Tortos et al., } \\
\text { 2008) }\end{array}$ \\
\hline Antidepressants & P-gp & $\begin{array}{l}\text { Amitriptyline, citalopram, desipramine, doxepin, } \\
\text { fluoxetine, imipramine, paroxetine, venlafaxine }\end{array}$ & In vitro permeability & $\left(O^{\prime} \text { Brien et al., 2012) }\right)^{144}$ \\
\hline Antihistamines & P-gp & Cetirizine, desloratadine, fexofenadine & In vitro permeability & (O'Brien et al., 2012) $)^{144}$ \\
\hline$\beta$-Blockers & P-gp & Talinolol & In vitro permeability & \\
\hline $\begin{array}{l}\text { Cardioactive } \\
\text { Agents }\end{array}$ & P-gp & Amiodarone, digoxin, diltiazem, quinidine, verapamil & In vitro permeability & $(\text { Caldwell et al., })^{164}$ \\
\hline Cytotoxics & P-gp, BCRP & $\begin{array}{l}\text { Daunorubicin, docetaxel, doxorubicin, etoposide, } \\
\text { mitoxantrone, paclitaxel, topotecan, vinblastine, } \\
\text { vincristine }\end{array}$ & In vitro permeability & $\begin{array}{l}\text { (Thomas and Coley, } \\
2003)^{145}\end{array}$ \\
\hline Gliptins & P-gp & $\begin{array}{l}\text { Sitagliptin, vildagliptin, saxagliptin, alogliptin, } \\
\text { linagliptin }\end{array}$ & In vitro permeability & $(\text { Scheen, 2010) })^{146}$ \\
\hline $\begin{array}{l}\mathrm{H}_{2} \text { receptor } \\
\text { antagonists }\end{array}$ & P-gp & Cimetidine, ranitidine & In vitro permeability & $\begin{array}{l}\text { (Neuvonen and Olkkola, } \\
\text { 1984; Dudley and } \\
\text { Brown, 1996) }\end{array}$ \\
\hline $\begin{array}{l}\text { HIV Protease } \\
\text { Inhibitors }\end{array}$ & P-gp & Indinavir, amprenavir, saquinavir, nelfinavir & mdr1a knockout & $\begin{array}{l}\text { (Choo et al., 2000; Kim, } \\
2002)^{147,148}\end{array}$ \\
\hline $\begin{array}{l}\text { Quinolone } \\
\text { Antibiotics }\end{array}$ & BCRP & Ciprofloxacin, ofloxacin, norfloxacin & $\begin{array}{l}\text { BCRP knockout, and in } \\
\text { vitro permeability }\end{array}$ & (Merino et al., 2005) \\
\hline Statins & P-gp & Pravastatin, atorvastatin, lovstatin, simvastatin & In vitro permeability & $(\text { Chen et al., 2005) })^{150}$ \\
\hline Steroids & P-gp & $\begin{array}{l}\text { Estrone, estradiol, ethynyl estradiol, norethindrone, } \\
\text { cortisol, aldosterone, dexamethasone }\end{array}$ & In vitro permeability & $\begin{array}{l}\text { (Ueda et al., 1992; Kim } \\
\text { and Benet, 2004) } \\
\text { 151,152 }\end{array}$ \\
\hline $\begin{array}{l}\text { Tyrosine Kinase } \\
\text { Inhibitors }\end{array}$ & BCRP & $\begin{array}{l}\text { Gefitinib, dasatinib, sorafenib, erlotinib, lapatinib, } \\
\text { imatinib, tandutinib }\end{array}$ & In vitro permeability & $($ Agarwal et al., 2011) \\
\hline
\end{tabular}


Table S6. Selected studies that tested effects of activated charcoal on pharmacokinetics and pharmacodynamics of drugs

\begin{tabular}{|c|c|c|c|c|}
\hline Drug Name & Charcoal Study & PK Effect & $\begin{array}{l}\text { Transporter } \\
\text { involved }\end{array}$ & Reference \\
\hline Amitriptyline & $\begin{array}{l}\text { Multiple charcoal doses in } \\
\text { humans }\end{array}$ & $\begin{array}{l}\text { multiple doses of charcoal reduced } \mathrm{T} 1 / 2 \\
\text { and increased elimination of drug and its } \\
\mathrm{N} \text {-demethyl metabolite }\end{array}$ & P-gp & $\begin{array}{l}\text { (Karkkainen and } \\
\text { Neuvonen, 1986) }\end{array}$ \\
\hline Amlodipine & $\begin{array}{l}25 \mathrm{~g} \text { treatment at different } \\
\text { time points in humans }\end{array}$ & $\begin{array}{l}99 \%, 49 \%, \text { and } 15 \% \text { reduction of AUC } \\
\text { at immediate, } 2 \text {, and } 5 \mathrm{~h} \text { after drug dose }\end{array}$ & P-gp & $\begin{array}{l}\text { (Laine et al., 1997a; } \\
\text { Kuzuya et al., } \\
2003)^{156,157}\end{array}$ \\
\hline Apixaban & $\begin{array}{l}\text { Charcoal after oral and IV } \\
\text { dose in rat, dog, and human }\end{array}$ & $\begin{array}{l}\text { Increased clearance, decreased } \mathrm{T} 1 / 2 \text {, } \\
\text { increased fecal excretion }\end{array}$ & P-gp, BCRP & This study \\
\hline Citalopram & $\begin{array}{l}\text { Charcoal dose } 0.5-4 \text { post drug } \\
\text { dose }\end{array}$ & $\begin{array}{l}\text { reduced absorption and AUC by } 51 \% \text {, } \\
\text { increased clearance by } 71 \% \text { and } F \\
\text { decreased by } 22 \%\end{array}$ & P-gp & (Friberg et al., 2005) $)^{161}$ \\
\hline Dapsone & $50 \mathrm{~g} \mathrm{x} 4$ doses in mans & $\mathrm{T} 1 / 2$ reduced by $50 \%$ & No & $\begin{array}{l}\text { (Neuvonen et al., } \\
1980)^{162}\end{array}$ \\
\hline Diazepam & $\begin{array}{l}25 \mathrm{~g} \text { dose } 30 \mathrm{~min} \text { after drug } \\
\text { dose }\end{array}$ & reduced absorption and AUC by $27 \%$ & & $\begin{array}{l}\text { (Lapatto-Reiniluoto et } \\
\text { al., 1999) }\end{array}$ \\
\hline
\end{tabular}




\begin{tabular}{|c|c|c|c|c|}
\hline Digoxin & $\begin{array}{l}\text { Charcoal dose of bile-duct } \\
\text { ligated rats, humans after IV }\end{array}$ & $\begin{array}{l}33.7 \% \text { of dose in stool collection } \\
\text { compared to } 13.4 \% \text { non-treated rats; } \\
\text { increased } \mathrm{Cl} \text { by } 47 \% \text {, decreased } \mathrm{T} 1 / 2 \text { and } \\
\text { MRT in humans }\end{array}$ & P-gp & $\begin{array}{l}\text { (Caldwell et al., 1980; } \\
\text { Lalonde et al., 1985; } \\
\text { Greiner et al., } \\
\text { 1999) }\end{array}$ \\
\hline Escitalopram & $\begin{array}{l}\text { single charcoal dose to } \\
\text { overdosed patients }\end{array}$ & $\begin{array}{l}\text { Reduced absorption by } 31 \% \text {, AUC, and } \\
\text { QT interval by } 35 \%\end{array}$ & P-gp & $\begin{array}{l}\text { (van Gorp et al., } \\
2012)^{168}\end{array}$ \\
\hline Furosemide & $\begin{array}{l}\text { Charcoal in rat of acute renal } \\
\text { failure after IV drug }\end{array}$ & No effects on serum concentrations & P-gp & $\begin{array}{l}\text { (Arimori and Nakano, } \\
\text { 1988a) }\end{array}$ \\
\hline GDC-0449 & $\begin{array}{l}\text { Charcoal dose in dogs after } \\
\text { PO and IV drug }\end{array}$ & $\begin{array}{l}\text { More rapid decrease in drug } \\
\text { concentrations }\end{array}$ & P-gp, BCRP & (Wong et al., 2010) ${ }^{128}$ \\
\hline Moxifloxacin & $\begin{array}{l}10 \mathrm{~g} \text { charcoal at } 15 \mathrm{~min}, 2,4, \\
8 \mathrm{~h} \text { after IV and PO dosed } \\
\text { drug in healthy subjects }\end{array}$ & $\begin{array}{l}\text { Reduced AUC (Cmax) by } 15-20 \% \text { after } \\
\text { IV and } 80 \% \text { after PO, F decreased }\end{array}$ & BCRP & $(\text { Stass et al., 2005) })^{48}$ \\
\hline $\begin{array}{l}\mathrm{N}- \\
\text { acetylcysteine }\end{array}$ & $\begin{array}{l}\text { treatment with charcoal } \\
\text { followed by oral drug in } \\
\text { humans }\end{array}$ & $\begin{array}{l}\text { Reduced AUC by by } 39 \% \text { and peak level } \\
\text { by } 29 \%\end{array}$ & No & (Ekins et al., 1987) ${ }^{172}$ \\
\hline Oxcarbazepine & $\begin{array}{l}20 \mathrm{~g} \text { repeated doses } 12 \mathrm{~h} \text { after } \\
\text { drug dose in humans }\end{array}$ & $\begin{array}{l}\text { Reduced AUC and T1/2 by } 39-44 \% \text {, } \\
\text { increased elimination }\end{array}$ & & $\begin{array}{l}\text { (Keranen et al., } \\
2010)^{171}\end{array}$ \\
\hline
\end{tabular}




\begin{tabular}{|c|c|c|c|c|}
\hline Phenobarbital & $\begin{array}{l}\text { Multiple dose after IV dose of } \\
\text { drug in humans }\end{array}$ & Increased $52-80 \%$ nonrenal clearance & P-gp & $\begin{array}{l}\text { (Berg et al., 1982; } \\
\text { Luna-Tortos et al., } \\
2008)^{173,143}\end{array}$ \\
\hline Phenytoin & $\begin{array}{l}\text { Treatment of rats IV dosed } \\
\text { drug } 50 \mathrm{mg} / \mathrm{kg}\end{array}$ & AUC and $\mathrm{T} 1 / 2$ reduced to $75-77 \%$ & P-gp & $\begin{array}{l}\text { (Arimori and Nakano, } \\
\text { 1987; Luna-Tortos et } \\
\text { al., 2008) }\end{array}$ \\
\hline Pholcodine & $\begin{array}{l}25 \mathrm{~g} \text { treatment at different } \\
\text { time points in humans }\end{array}$ & $\begin{array}{l}91 \%(77 \%, 85 \%)), 26 \%(23 \%, 28 \%) \text {, and } \\
17 \% \text { reduction of AUC (Cmax, urinary } \\
\text { excretion) at immediate, } 2 \text {, and } 5 \text { h after } \\
\text { drug dose }\end{array}$ & P-gp & (Laine et al., 1997c) ${ }^{175}$ \\
\hline Pindolol & $\begin{array}{l}\text { treatment } 5 \text { min after drug } \\
\text { dose in humans }\end{array}$ & Reduced absorption by $99 \%$ & P-gp & $\begin{array}{l}\text { (Neuvonen and } \\
\text { Olkkola, 1984) }\end{array}$ \\
\hline Procainamide & $\begin{array}{l}\text { Treatment of rats with acute } \\
\text { renal failure }\end{array}$ & $\begin{array}{l}\text { Reduced serum drug level in ARF rats but } \\
\text { not in normal rats }\end{array}$ & $\begin{array}{l}\text { P-gp } \\
\text { inhibitor }\end{array}$ & $\begin{array}{l}\text { (Arimori and Nakano, } \\
\text { 1988b; Arimori et al., } \\
\text { 1990) } \\
{ }^{176,177}\end{array}$ \\
\hline $\begin{array}{l}\text { Quinidine / } \\
\text { Qunine }\end{array}$ & $\begin{array}{l}15 \mathrm{~g} \text { charcoal in IV dosed } \\
\text { rabbits }\end{array}$ & $\begin{array}{l}\text { Increased CL, reduced T1/2, AUC, MRT } \\
\text { on quinidine but no effect on quinine }\end{array}$ & P-gp & (Hasan et al., 1990) $^{181}$ \\
\hline Rifampicin & $\begin{array}{l}15 \mathrm{~g} \text { dose together with drug } \\
\text { in humans }\end{array}$ & $\begin{array}{l}\text { Led to } 1.2 \% \text { urinary excretion lower } \\
\text { plateau indicative of reduced absorption }\end{array}$ & No & $\begin{array}{l}\text { (Orisakwe et al., } \\
1996)^{182}\end{array}$ \\
\hline Salicylate & $\begin{array}{l}\text { single and multiple charcoal } \\
\text { dose in rats or humans }\end{array}$ & $\begin{array}{l}\text { No effect in rats; Reduced urine recovery } \\
\text { from } 91 \% \text { to } 68.3,65.9 \text {, and } 49.2 \% \text { for } 1 \text {, } \\
2 \text { and } 3 \text { dose of charcoal }\end{array}$ & No & $\begin{array}{l}\text { (Barone et al., 1988; } \\
\text { Mayer et al., } \\
1992)^{183,184}\end{array}$ \\
\hline
\end{tabular}




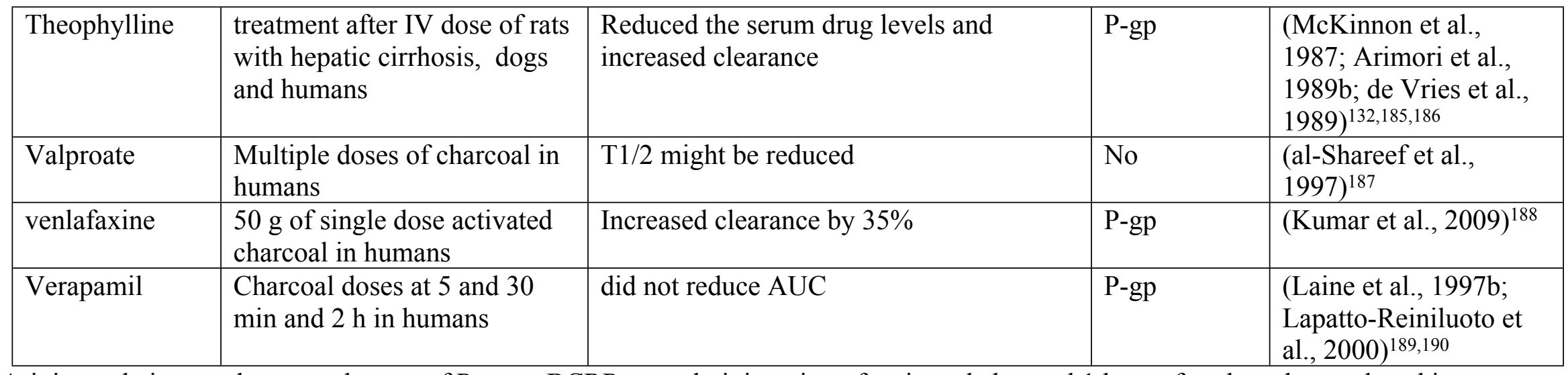

Aripiprazole is not a known substrate of P-gp or BCRP, yet administration of activated charcoal 1 hour after drug dose reduced its Cmax and AUC by approximately $50 \%$ with an increase of clearance of $100 \%$ in humans. 
Figure S1. Chemical structures used in Table S5

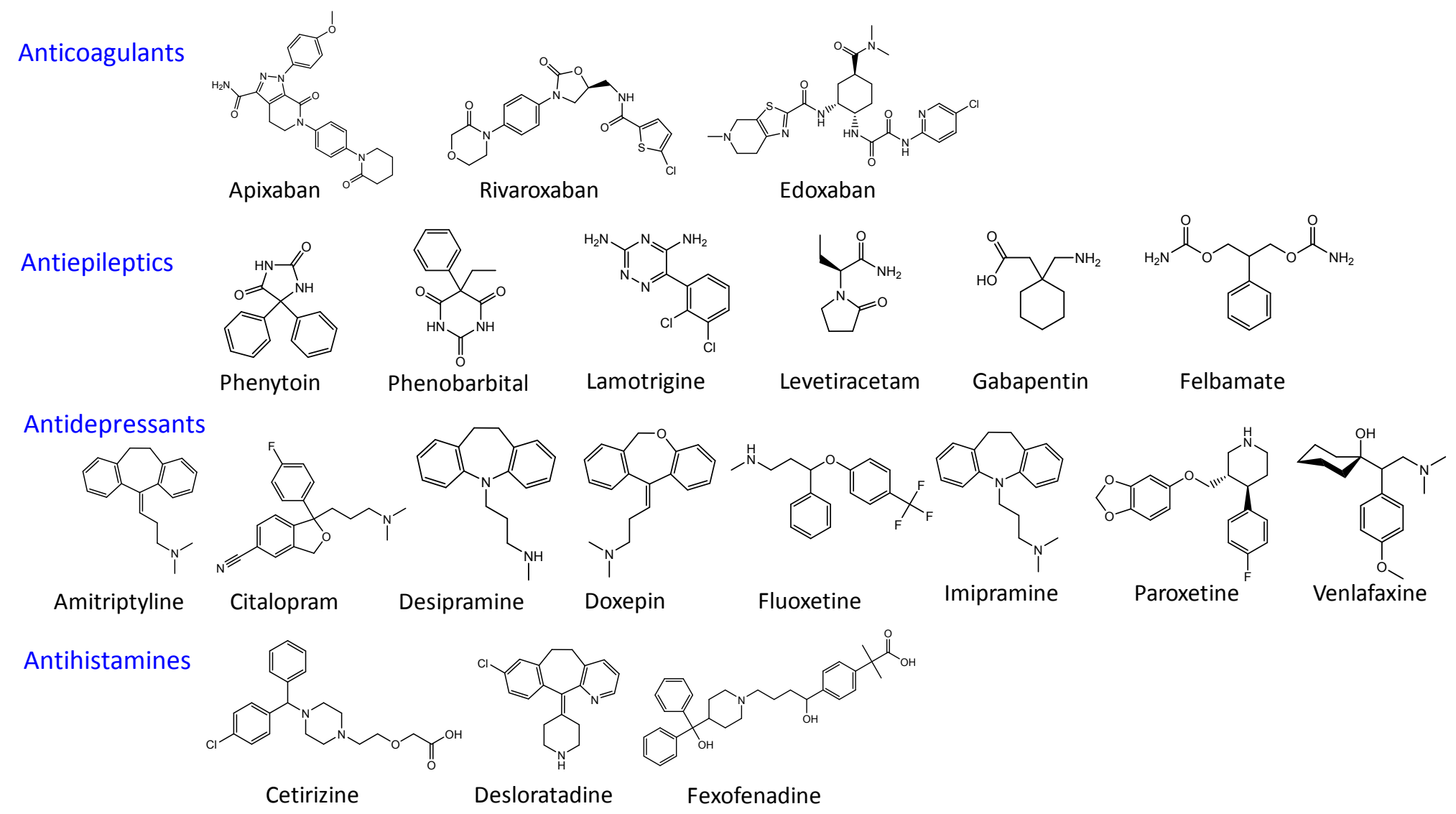


Fig S1 Continued

$\beta$-Blockers<smiles></smiles>

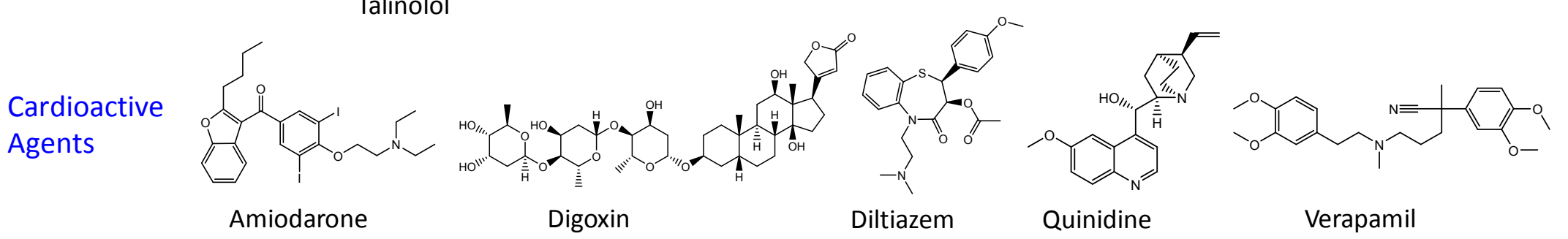

Cytotoxics

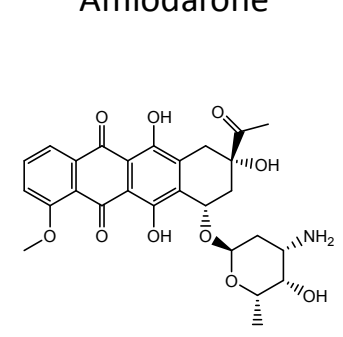

Daunorubicin

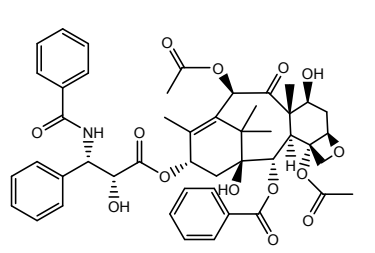

Paclitaxel

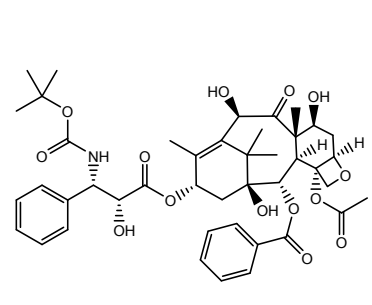

Docetaxel

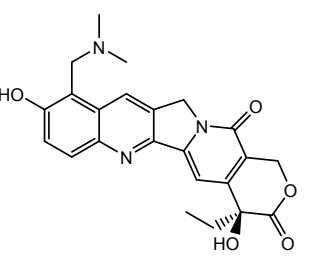

Topotecan

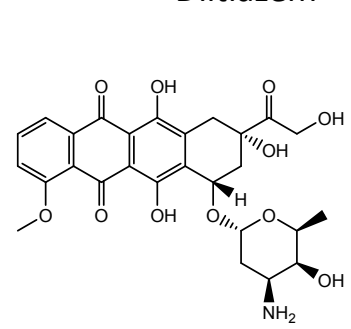

Doxorubicin

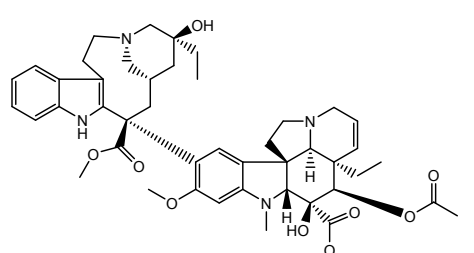

Vinblastine

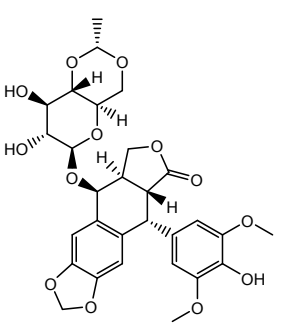

Etoposide

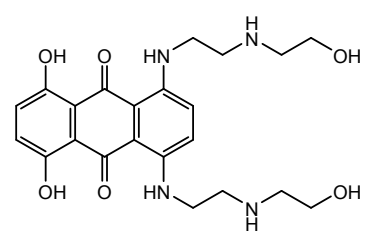

Mitoxantrone

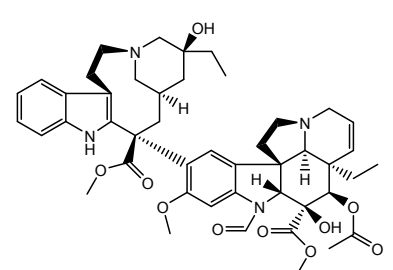

Vincristine 
Fig S1 Continued

\section{Gliptins}

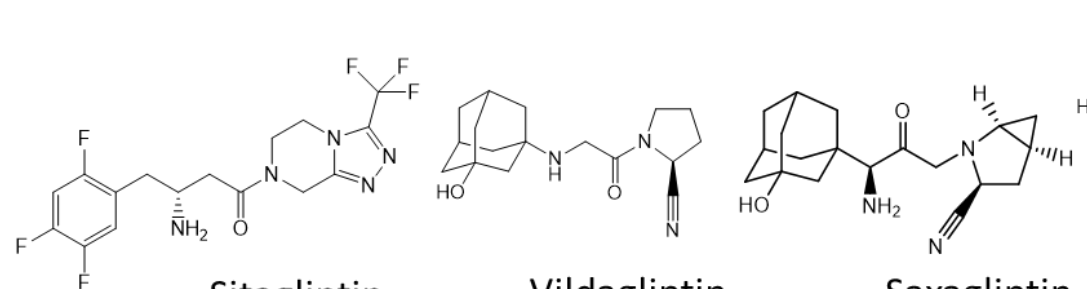

Sitagliptin

Vildagliptin

Saxagliptin

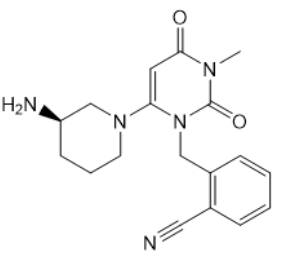

Alogliptin

$\mathrm{H}_{2}$ receptor antagonists
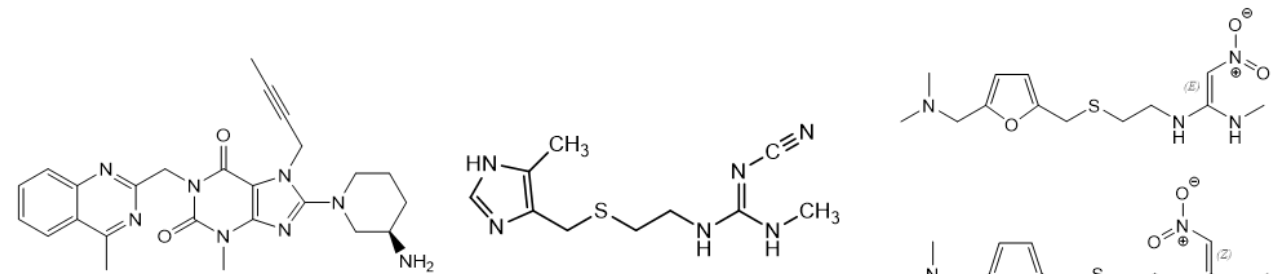

Linagliptin
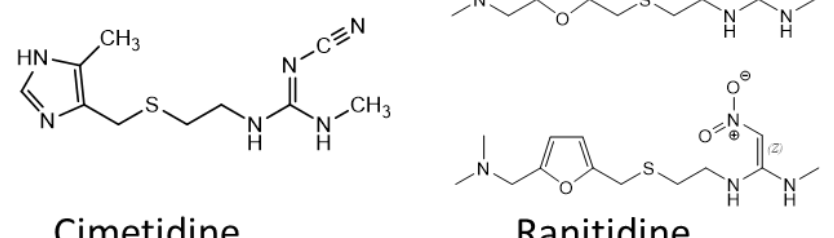

HIV Protease Inhibitors

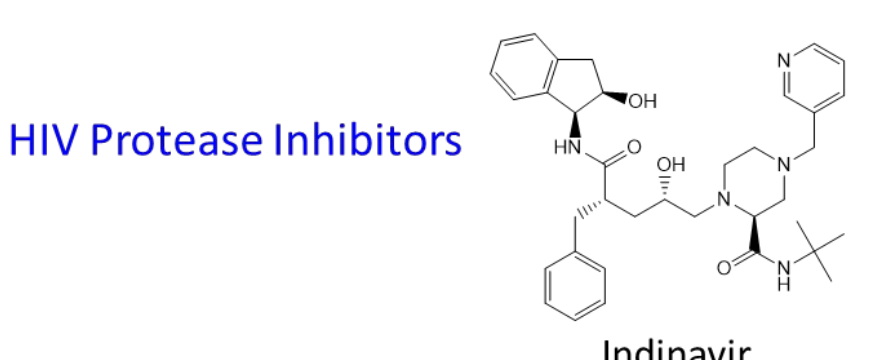

Cimetidine

Ranitidine
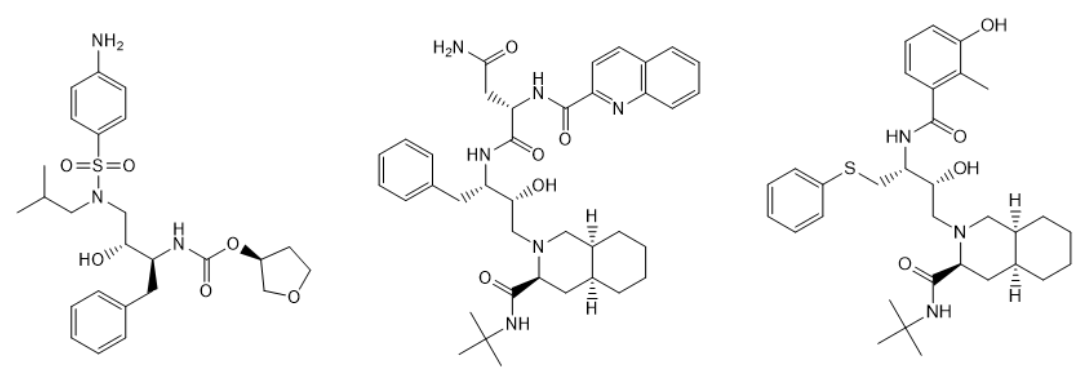

Amprenavir

Saquinavir

Nelfinavir

Quinolone Antibiotics

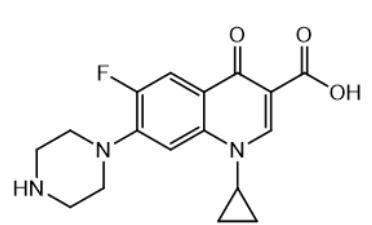

Ciprofloxacin

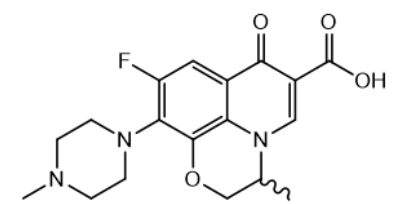

Ofloxacin

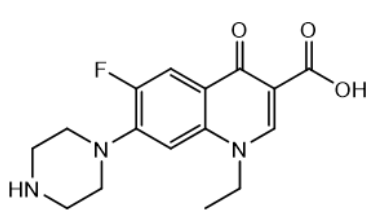

Norfloxacin 
Fig S1 Continued

Statins

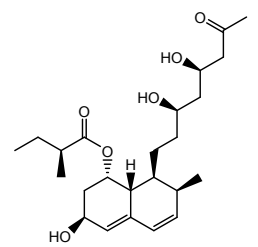

Pravastatin

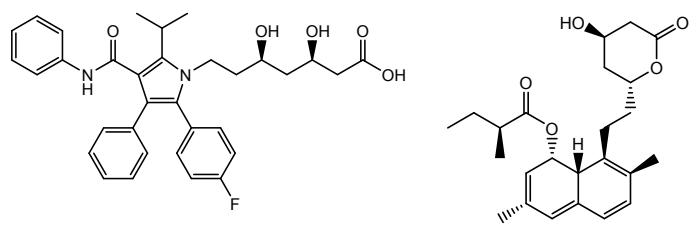

Atorvastatin

Lovastatin

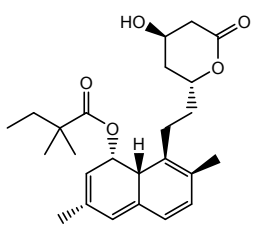

Simvastatin
Steroids

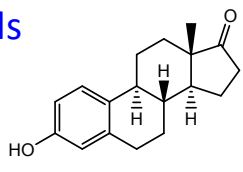

Estrone

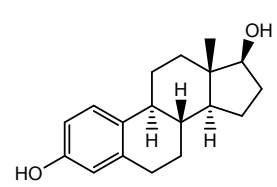

Estradiol

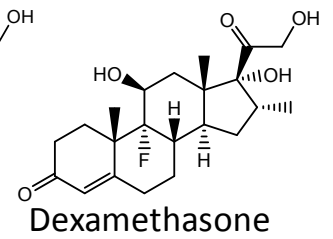

Tyrosine Kinase Inhibitors

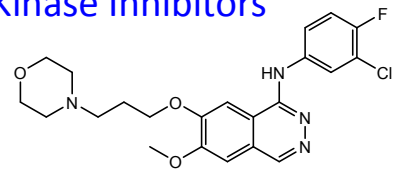

Gefitinib

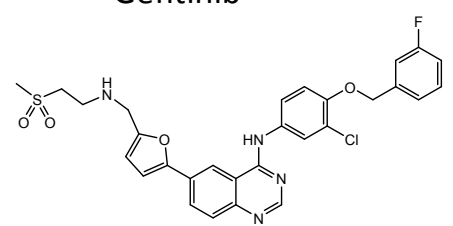

Lapatinib

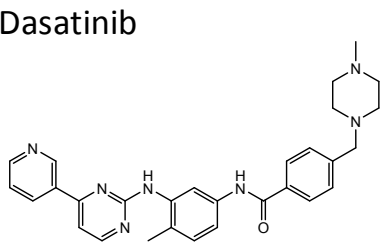

Imatinib
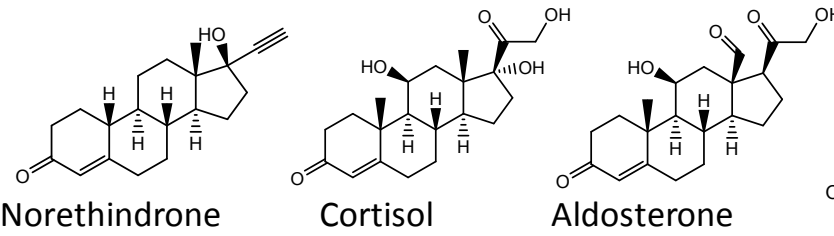

Norethindrone

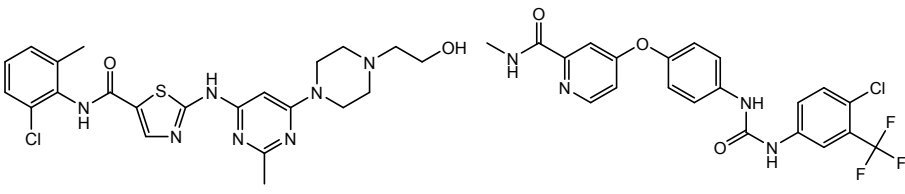

Sorafenib<smiles>C#Cc1cccc(NC2NCNc3cc(OCCOC)c(OCCOC)cc32)c1</smiles>

Erlotinib
Tandutinib

S15 
Additional References:

142) Loscher, W.; Potschka, H. Blood-brain barrier active efflux transporters: ATP-binding cassette gene family. NeuroRx. 2005, 2, 86-98.

143) Luna-Tortos, C.; Fedrowitz, M.; Loscher, W. Several major antiepileptic drugs are substrates for human Pglycoprotein. Neuropharmacology 2008, 55, 1364-1375.

144) O'Brien, F. E.; Dinan, T. G.; Griffin, B. T.; Cryan, J. F. Interactions between antidepressants and P-glycoprotein at the blood-brain barrier: clinical significance of in vitro and in vivo findings. Br. J. Pharmacol. 2012, 165, $289-312$.

145) Thomas, H.; Coley, H. M. Overcoming multidrug resistance in cancer: an update on the clinical strategy of inhibiting p-glycoprotein. Cancer Control 2003, 10, 159-165.

146) Scheen, A. J. Pharmacokinetics of dipeptidylpeptidase-4 inhibitors. Diabetes Obes. Metab. 2010, 12, 648-658.

147) Choo, E. F.; Leake, B.; Wandel, C.; Imamura, H.; Wood, A. J.; Wilkinson, G. R.; Kim, R. B. Pharmacological inhibition of P-glycoprotein transport enhances the distribution of HIV-1 protease inhibitors into brain and testes. Drug Metab. Dispos. 2000, 28, 655-660.

148) Kim. R. B. Drugs as P-glycoprotein substrates, inhibitors, and inducers. Drug Metab. Rev. 2002, $34,47-54$.

149) Merino, G.; Jonker, J. W.; Wagenaar, E.; van Herwaarden, A. E.; Schinkel, A. H. The breast cancer resistance protein (BCRP/ABCG2) affects pharmacokinetics, hepatobiliary excretion, and milk secretion of the antibiotic nitrofurantoin. Mol.

Pharmacol. 2005, 67, 1758-1764.

150) Chen, C.; Mireles, R. J.; Campbell, S. D.; Lin, J.; Mills, J. B.; Xu, J. J.; Smolarek, T. A. Differential interaction of 3hydroxy-3-methylglutaryl-coa reductase inhibitors with ABCB1, ABCC2, and OATP1B1. Drug Metab. Dispos. 2005, 33, 537-546.

151) Ueda, K.; Okamura, N.; Hirai, M.; Tanigawara, Y.; Saeki, T.; Kioka, N.; Komano, T.; Hori, R. Human P-glycoprotein transports cortisol, aldosterone, and dexamethasone, but not progesterone. J. Biol. Chem. 1992, 267, 24248-24252.

152) Kim, W. Y.; Benet, L. Z. P-glycoprotein (P-gp/MDR1)-mediated efflux of sex-steroid hormones and modulation of Pgp expression in vitro. Pharm. Res. 2004, 21, 1284-1293.

153) Eyer, F.; Jung, N.; Neuberger, H.; Schulz, R.; Steiner, K.; Ladstetter, B.; Poethko, T.; Henke, J.; Zilker, T. Enteral exsorption of acetaminophen after intravenous injection in rats: influence of activated charcoal on this clearance path. Basic Clin. Pharmacol. Toxicol. 2007, 101, 163-171.

154) Mullins, M.; Froelke, B. R.; Rivera, M. R. Effect of delayed activated charcoal on acetaminophen concentration after simulated overdose of oxycodone and acetaminophen. Clin. Toxicol. 2009, 47, 112-115.

155) Karkkainen, S.; Neuvonen, P. J. Pharmacokinetics of amitriptyline influenced by oral charcoal and urine pH. Int. J. Clin. Pharmacol. Ther. Toxicol. 1986, 24, 326-332. 
156) Laine, K.; Kivisto, K. T.; Laakso, I.; Neuvonen, P. J. Prevention of amlodipine absorption by activated charcoal: effect of delay in charcoal administration. Br. J. Clin. Pharmacol. 1997a, 43, $29-33$.

157) Kuzuya, T.; Kobayashi, T.; Moriyama, N.; Nagasaka, T.; Yokoyama, I.; Uchida, K.; Nakao, A.; Nabeshima, T. Amlodipine, but not MDR1 polymorphisms, alters the pharmacokinetics of cyclosporine A in Japanese kidney transplant recipients. Transplantation 2003, 76, 865-868.

158) Eyer, F.; Jung, N.; Neuberger, H.; Witte, A.; Poethko, T.; Henke, J.; Zilker, T. Seromucosal transport of intravenously administered carbamazepine is not enhanced by oral doses of activated charcoal in rats. Basic Clin. Pharmacol. Toxicol. 2008, 102, 337-346.

159) Neuvonen, P. J.; Olkkola, K. T. Activated charcoal and syrup of ipecac in prevention of cimetidine and pindolol absorption in man after administration of metoclopramide as an antiemetic agent. J. Toxicol. Clin. Toxicol. 1984, 22, $103-114$.

160) Dudley, A. J., Brown, C. D. Mediation of cimetidine secretion by P-glycoprotein and a novel H(+)-coupled mechanism in cultured renal epithelial monolayers of LLC-PK1 cells. Br. J. Pharmacol. 1996, 117, 1139-1144.

161) Friberg, L. E.; Isbister, G. K.; Hackett, L. P.; Duffull, S. B. The population pharmacokinetics of citalopram after deliberate self-poisoning: a Bayesian approach. J. Pharmacokinet. Pharmacodyn. 2005, 32, 571-605.

162) Neuvonen, P. J.; Elonen, E.; Mattila, M. J. Oral activated charcoal and dapsone elimination. Clin. Pharmacol. Ther. $1980,27,823-827$.

163) Lapatto-Reiniluoto, O.; Kivisto, K. T.; Neuvonen, P. J. Effect of activated charcoal alone or given after gastric lavage in reducing the absorption of diazepam, ibuprofen and citalopram. Br. J. Clin. Pharmacol. 1999, 48, 148-153.

164) Caldwell, J. H.; Caldwell, P. B.; Murphy, J. W.; Beachler, C. W. Intestinal secretion of digoxin in the rat. Augmentation by feeding activated charcoal. Naunyn Schmiedebergs Arch Pharmacol. 1980, 312, 271-275.

165) Lalonde, R. L.; Deshpande, R.; Hamilton, P. P.; McLean, W. M.; Greenway, D. C. Acceleration of digoxin clearance by activated charcoal. Clin. Pharmacol. Ther. 1985, 37, 367-371.

166) Arimori, K.; Kawano, H.; Nakano, M. Gastrointestinal dialysis of disopyramide in healthy subjects. Int. J. Clin. Pharmacol. Ther. Toxicol. 1989a, 27, 280-284.

167) Arimori, K.; Nakano, M. Study on transport of disopyramide into the intestinal lumen aimed at gastrointestinal dialysis by activated charcoal in rats. J. Pharm. Pharmacol. 1989, 41, 445-449.

168) van Gorp, F.; Duffull, S.; Hackett, L. P.; Isbister, G. K. Population pharmacokinetics and pharmacodynamics of escitalopram in overdose and the effect of activated charcoal. Br. J. Clin. Pharmacol. 2012, 73, 402-410.

169) Laine, K.; Kivisto, K. T.; Pelttari, S.; Neuvonen, P. J. The effect of activated charcoal on the absorption of fluoxetine, with special reference to delayed charcoal administration. Pharmacol. Toxicol. 1996, 79, 270-273. 
170) Sergio, G. C.; Felix, G. M.; Luis, J. V. Activated charcoal to prevent irinotecan-induced diarrhea in children. Pediatr. Blood Cancer 2008, 51, 49-52.

171) Keranen, T.; Sorri, A.; Moilanen, E.; Ylitalo, P. Effects of charcoal on the absorption and elimination of the antiepileptic drugs lamotrigine and oxcarbazepine. Arzneimittelforschung. 2010, 60, 421-426.

172) Ekins, B. R.; Ford, D. C.; Thompson, M. I.; Bridges, R. R.; Rollins, D. E.; Jenkins, R. D. The effect of activated charcoal on $\mathrm{N}$-acetylcysteine absorption in normal subjects. Am. J. Emerg. Med. 1987, 5, 483-487.

173) Berg, M. J.; Berlinger, W. G.; Goldberg, M. J.; Spector, R.; Johnson, G. F. Acceleration of the body clearance of phenobarbital by oral activated charcoal. N. Engl. J. Med. 1982, 307, 642-644.

174) Arimori, K.; Nakano, M. The intestinal dialysis of intravenously administered phenytoin by oral activated charcoal in rats. J. Pharmacobiodyn. 1987, 10, 157-165.

175) Laine, K.; Kivisto, K. T.; Ojala-Karlsson, P.; Neuvonen, P. J. Effect of activated charcoal on the pharmacokinetics of pholcodine, with special reference to delayed charcoal ingestion. Ther. Drug. Monit. 1997c, 19, 46-50.

176) Arimori, K.; Nakano, M. Transport of procainamide and N-acetylprocainamide from blood into the intestinal lumen and intestinal dialysis by oral activated charcoal in rats with acute renal failure. J. Pharmacobiodyn. 1988b, 11, 504-511.

177) Arimori, K.; Hashimoto, Y.; Nakano, M. Comparison of transport of procainamide and N-acetylprocainamide from blood into the intestinal lumen with that into the peritoneal cavity in rats. Chem. Pharm. Bull. 1990, 38, $2050-2052$.

178) Yang, J. J.; Kim, K. J.; Lee, V. H. Role of P-glycoprotein in restricting propranolol transport in cultured rabbit conjunctival epithelial cell layers. Pharm. Res. 2000, 17, 533-538.

179) Boulton, D. W.; DeVane, C. L.; Liston, H. L.; Markowitz, J. S. In vitro P-glycoprotein affinity for atypical and conventional antipsychotics. Life Sci. 2002, 71, 163-169.

180) Isbister, G. K.; Friberg, L. E.; Hackett, L. P.; Duffull, S. B. Pharmacokinetics of quetiapine in overdose and the effect of activated charcoal. Clin. Pharmacol. Ther. 2007, 81, 821-827.

181) Hasan, M. M.; Hassan, M. A. Rawashdeh, N. M. Effect of oral activated charcoal on the pharmacokinetics of quinidine and quinine administered intravenously to rabbits. Pharmacol. Toxicol. 1990, 67, 73-76.

182) Orisakwe, O. E.; Dioka, C. E.; Okpogba, A. N.; Orish, C. N.; Ofoefule, S. I. Effect of activated charcoal on rifampicin absorption in man. Tokai J. Exp. Clin. Med. 1996, 21, 51-54.

183) Barone, J. A.; Raia, J. J.; Huang, Y. C. Evaluation of the effects of multiple-dose activated charcoal on the absorption of orally administered salicylate in a simulated toxic ingestion model. Ann. Emerg. Med. 1988, 17, 34-37.

184) Mayer, A. L.; Sitar. D. S.; Tenenbein, M. Multiple-dose charcoal and whole-bowel irrigation do not increase clearance of absorbed salicylate. Arch. Intern. Med. 1992, 152, 393-396. 
185) McKinnon, R. S.; Desmond, P. V.; Harman, P. J.; Kamm, M.; Ghabrial, H.; Martin, C. J.; Mashford, M. L. Studies on the mechanisms of action of activated charcoal on theophylline pharmacokinetics. J. Pharm. Pharmacol. 1987, 39, 522-525.

186) de Vries, M. H.; Rademaker, C. M.; Geerlings C.; Van Dijk, A.; Noordhoek, J. Pharmacokinetic modelling of the effect of activated charcoal on the intestinal secretion of theophylline, using the isolated vascularly perfused rat small intestine. $J$. Pharm. Pharmacol. 1989, 41, 528-533.

187) al-Shareef, A.; Buss, D. C.; Shetty, H. G.; Ali, N.; Routledge, P. A. The effect of repeated-dose activated charcoal on the pharmacokinetics of sodium valproate in healthy volunteers. Br. J. Clin. Pharmacol. 1997, 43, 109-111.

188) Kumar, V. V.; Oscarsson, S.; Friberg, L. E.; Isbister, G. K.; Hackett, L. P.; Duffull, S. B. The effect of decontamination procedures on the pharmacokinetics of venlafaxine in overdose. Clin. Pharmacol. Ther. 2009, 86, 403-410.

189) Laine, K.; Kivisto, K. T.; Neuvonen, P. J. Effect of delayed administration of activated charcoal on the absorption of conventional and slow-release verapamil. J. Toxicol. Clin. Toxicol. 1997b, 35, 263-268.

190) Lapatto-Reiniluoto, O.; Kivisto, K. T.; Neuvonen, P. J.; Gastric decontamination performed 5 min after the ingestion of temazepam, verapamil and moclobemide: charcoal is superior to lavage. Br. J. Clin. Pharmacol. 2000, 49, $274-278$. 